\title{
How we proposed the spin transistor
}

\author{
Spintronic devices, which exploit the spin of electrons for information processing and storage, are a key emerging \\ technology in electronics. Supriyo Datta explains how emulating optical phenomena inspired his prediction of the \\ spin transistor.
}

\section{Supriyo Datta}

n 1990, Biswajit Das and I proposed an electronic device similar to wellknown optical devices by exploiting the similarities between the spin of an electron and the polarization of a photon (Fig. 1). The inspiration for this was an optical device that consisted of a polarizer and an analyser set at $90^{\circ}$ to each other. In this initial configuration, the transmission of light would be minimal. However, between the polarizer and analyser was an electrooptic material that allowed an external voltage to rotate the polarization of the light as it propagated. If the rotation was $90^{\circ}$, transmission would be perfect.

But what would an electronic analogue look like? The proposal was to use a device that looks just like a commercial field-effect transistor (FET), but with contacts that are magnetic. One magnetic contact (the source) injects electrons whose spins are aligned with its magnetization, just as a polarizer transmits photons with a specific polarization. Likewise, the other magnetic contact (the drain) acts as an analyser and lets through electrons with appropriately aligned spin. If the source contact injects up-spin electrons and the drain contact detects down-spin electrons, then the transmission should be a minimum. If the spin could be rotated as it traverses the channel connecting source to drain, the transmission could be increased, just like the optical device.

Note here an interesting difference. To block photons, we need a polarizer and analyser at $90^{\circ}$, but for electrons they need to be at $180^{\circ}$. This is a fundamental difference: photon polarization is described by vectors whereas electron spin is described by spinors.

The next challenge was to rotate the spin. One way would be to use a magnetic field in the $x$ direction so that the spin rotates around it in the $y z$ plane. Another way would be to use an electric field, provided the channel exhibits spin-orbit interaction, which is a well-known relativistic effect that makes a $y$-directed electric field at the interface look like an effective $x$-directed magnetic field to an electron with a $z$-directed momentum. But back in 1990, there was no experimental evidence that a gate voltage could be used to control the spin-orbit interaction and it took until 1997 for it to be first demonstrated by Junsaku Nitta and colleagues at the NTT Basic Research Laboratories in Japan.

As for the injection and detection of polarized spins with magnetic contacts, Mark Johnson and Robert Silsbee at Cornell University had already demonstrated the possibility in 1985. But that involved metallic channels. Semiconducting channels proved to be much harder and it took until 2001 before researchers were able to overcome the formidable challenges involved. Finally, Hyun Cheol Koo and colleagues at the Korea Institute of Science and Technology, working with Johnson, who was then at the US Naval Research Laboratory, demonstrated one version of the device in 2009 and then a different version in 2015

This device is often called a spin transistor, possibly because a gate voltage changes its resistance from a high value $(\mathrm{OFF})$ to a low value $(\mathrm{ON})$, like a real transistor. But a typical FET has an OFF/ON resistance ratio of about $10^{5}$. By contrast, the spin transistor can at best reduce the current a little bit, partly because polarizers and analysers are far from ideal and partly because scattering processes in solids can randomize spins quite strongly depending on the temperature and the channel length. Alternative designs have been proposed to overcome this limitation.

One spintronic device that has proved to be of practical use in memory applications consists of two magnetic contacts spaced by a very thin layer, which could either be a metal (creating a spin valve) or an insulator (creating a magnetic tunnel junction; MTJ).

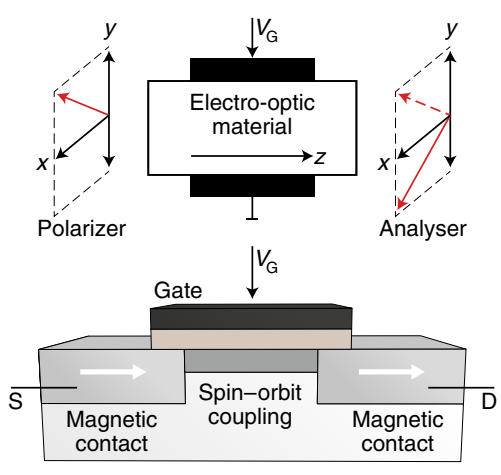

Fig. 1 | Photon polarization and electron spin. Concept of spin transistor illustrating the analogy between photon polarization (top) and electron spin (bottom). $V_{G}$ gate voltage; $S$, source; $D$, drain.

Both these devices were known before 1990, but, in retrospect, they can be viewed as polarizer-analyser devices with a very short channel, so that the effects of scattering are not that severe.

For a long time, researchers looked for a device to replace the FET. But it seems more practical to find ways to complement the FET. For example, small magnets are unstable, making the resistance of an MTJ fluctuate with time. It has been argued that such a stochastic MTJ could be combined with FETs to build a probabilistic bit or a p-bit: a three-terminal device whose output alternates between ' 0 ' and ' 1 ' when the input is weak, but locks into ' 0 ' for large negative input and to ' 1 ' for large positive input. This controlled stochastic behaviour with a single compact unit seems difficult to achieve with FETs alone.

\section{Supriyo Datta}

School of Electrical and Computer Engineering, Purdue University, West Lafayette, IN, USA. e-mail:datta@purdue.edu

Published online: 12 November 2018 https://doi.org/10.1038/s41928-018-0163-4 